\title{
The theorem of the complement for a quasi subanalytic set
}

\author{
by \\ AbDelhafed ElKhadiri (Kénitra) \\ Dedicated to Professor Jean-Claude Tougeron
}

\begin{abstract}
Let $X \subset\left(\mathbb{R}^{n}, 0\right)$ be a germ of a set at the origin. We suppose $X$ is described by a subalgebra, $C_{n}(M)$, of the algebra of germs of $C^{\infty}$ functions at the origin (see 2.1). This algebra is quasianalytic. We show that the germ $X$ has almost all the properties of germs of semianalytic sets. Moreover, we study the projections of such germs and prove a version of Gabrielov's theorem.
\end{abstract}

Introduction. The aim of this paper is to study germs, at the origin in $\mathbb{R}^{n}$, of some sets defined as finite unions of sets of the form

$$
\left\{x \mid \varphi_{0}(x)=0, \varphi_{1}(x)>0, \ldots, \varphi_{q}(x)>0\right\},
$$

where $\varphi_{0}, \ldots, \varphi_{q}$ are elements of a subalgebra, say $C_{n}(M)$, of the algebra of $C^{\infty}$ germs at the origin. We will call those germs quasi semianalytic germs and their projections quasi subanalytic. We suppose that our algebra contains the germs of real-analytic functions at the origin and is quasianalytic, that is, if $f \in C_{n}(M)$ is such that its Taylor series at the origin, say $T_{0} f$, is zero, then the germ $f$ is null. It is well known [3] that the Weierstrass division theorem does not hold in $C_{n}(M)$, and we do not know if this algebra is noetherian or not; so we cannot completely follow the methods used in the classical case, i.e. when $C_{n}(M)$ is the algebra of analytic germs, to study quasi semianalytic germs and their projections.

By using elementary blowings-up of $\mathbb{R}^{n}$ with smooth center, we can prove that by a finite number of blowings-up we can transform any $f \in C_{n}(M)$, modulo a product by an invertible element in $C_{n}(M)$, to a monomial (Proposition 7$)$. This implies that $C_{n}(M)$ is topologically noetherian, that is, every decreasing sequence of germs is stationary. This property is enough for us

2000 Mathematics Subject Classification: Primary 32Bxx, 14Pxx; Secondary 26E10.

Key words and phrases: quasianalytic functions, subanalytic and semianalytic sets, Gabrielov's theorem.

Recherche menée dans le cadre du projet PARS MI 33. 
to extend some well known properties of semianalytic germs (stratification, locally finite number of connected components, ...) to the quasi semianalytic germs. We also prove that the closure and each connected component of a quasi semianalytic germ are quasi semianalytic. The Tarski-Seidenberg theorem is not true in this class of germs, so in Section 8 we study the quasi subanalytic germs. The main results are Theorem 7 , which gives a uniform bound of the number of connected components of the fibers of a projection restricted to a bounded quasi subanalytic set, and Lemma 7, which shows that the dimension of quasi semianalytic germs is well behaved.

Finally, we prove the complement theorem for quasi subanalytic germs. This theorem is also proved in [10] by J.-P. Rolin, P. Speissegger and A. J. Wilkie. Our approach is different. The normalization algorithm used in Section 2 of [10] is more complicated than the proof of our Proposition 7, and our way of introducing the class of functions is more convenient. We also have a theory of quasi semianalytic germs (Theorems 5,6). Moreover, we prove the Łojasiewicz inequalities for functions in this class in the same way that was used in [11] for the Gevrey class.

The author thanks Professor A. J. Wilkie for his comments.

1. Background. Let $n$ be a positive integer, $\alpha=\left(\alpha_{1}, \ldots, \alpha_{n}\right) \in \mathbb{N}^{n}$, and $x=\left(x_{1}, \ldots, x_{n}\right)$ the canonical coordinates on $\mathbb{R}^{n}$.

We use the standard notations: $|\alpha|=\alpha_{1}+\ldots+\alpha_{n}, \alpha !=\alpha_{1} ! \ldots \alpha_{n} !, D^{\alpha}=$ $\partial^{|\alpha|} / \partial x_{1}^{\alpha_{1}} \ldots \partial x_{n}^{\alpha_{n}}$, and a preorder on $\mathbb{N}^{n}$ is defined by $\alpha=\left(\alpha_{1}, \ldots, \alpha_{n}\right) \leq$ $\beta=\left(\beta_{1}, \ldots, \beta_{n}\right) \Leftrightarrow \alpha_{i} \leq \beta_{i}, \forall i=1, \ldots, n$.

We say that a real function, $m$, of one real variable is $C^{\infty}$ for $t \gg 0$ if there is $b>0$ such that $m$ is $C^{\infty}$ in the interval $[b, \infty[$.

In all the following, $m$ will be a $C^{\infty}$ function for $t \gg 0$ with $m, m^{\prime}, m^{\prime \prime}$ $>0, \lim _{t \rightarrow \infty} m^{\prime}(t)=\infty$ and there is $\delta>0$ such that $m^{\prime \prime}(t) \leq \delta$ for $t \gg 0$. We put

$$
M(t)=e^{m(t)} .
$$

If $U \subset \mathbb{R}^{n}$ is an open subset, $\mathcal{E}(U)$ denotes the algebra of $C^{\infty}$ functions on $U$.

\section{Functions of class $M$}

Definition 1. A function $f \in \mathcal{E}(U)$ is said to be in the class $M$ if for each compact $K \subset U$, there are $C_{K}, \varrho_{K}>0$ such that for all $x \in K$,

$$
\left|D^{\alpha} f(x)\right| \leq C_{K} \varrho_{K}^{|\alpha|} M(|\alpha|) \quad \text { for }|\alpha| \gg 0 .
$$

We let $C_{U}(M)$ be the collection of all $C^{\infty}$ functions on $U$ which are in the class $M$. 
REMARK 1. Let $M_{1}(t)=c r^{t} M(t)$, where $c, r>0$. We easily see that a function $f \in \mathcal{E}(U)$ is in the class $M$ if and only if $f$ is in the class $M_{1}$; hence the class does not change when $m(t)$ is replaced by $m(t)+a t+b, a, b \in \mathbb{R}$; so we will suppose in the following that $m(0)=0$.

In the following, if $m:\left[b, \infty\left[\rightarrow \mathbb{R}, m(b)=0, b \geq 0\right.\right.$ and $m, m^{\prime}, m^{\prime \prime}>0$ in the interval $[b, \infty[$, we still denote by $m$ the extension of $m$ to $[0, \infty[$ obtained by setting $m(t)=0$ if $t \leq b$. We see that this extension is convex.

Lemma 1. For all $j \in \mathbb{N}, j \gg 0$, there exist $C_{j}, \varrho_{j}>0$ with

$$
M(p+j) \leq C_{j} \varrho_{j}^{p} M(p), \quad \forall p \in \mathbb{N}, p \gg 0 .
$$

Proof. There exists $\theta \in] p, p+j\left[\right.$ such that $m(p+j)-m(p)=j m^{\prime}(\theta)$. Since $m^{\prime \prime} \leq \delta$, there exists $C>0$ with $m^{\prime}(t) \leq \delta t+C$. We have $m^{\prime}(\theta) \leq$ $m^{\prime}(p+j) \leq \delta p+(C+\delta j)$. Put $\varrho_{j}=e^{j \delta}$ and $C_{j}=e^{j(\delta j+C)}$; then $M(p+j) \leq$ $C_{j} \varrho_{j}^{p} M(p)$.

Lemma 2. $C_{U}(M)$ is an algebra, closed under differentiation.

Proof. Since $m$ is convex and $m(0)=0$, for $0 \leq j \leq n$ we have

$$
m(n-j) \leq \frac{n-j}{n} m(n), \quad m(j) \leq \frac{j}{n} m(n)
$$

hence $m(j)+m(n-j) \leq m(n)$, i.e. $M(n-j) M(j) \leq M(n)$. Using this inequality and the Leibniz formula, we deduce the first statement of the lemma. The second statement follows immediately from Lemma 1.

The following theorem gives a one-dimensional characterization of functions in the class $M$ and can be considered as an extension of a result in [4].

Let $\Omega$ be an open subset of the sphere $S^{n-1} \subset \mathbb{R}^{n}(n>1)$ and $f \in \mathcal{E}(U)$. We suppose that the following condition on $f$ is satisfied:

(*) For each $\xi \in \Omega$ and each compact subset $K \subset U$, there exists a constant $C_{K, \xi}>0$ such that

$$
\left|\frac{d^{m}}{d t^{m}} f(x+t \xi)_{\mid t=0}\right| \leq C_{K, \xi} M(m) \quad \forall x \in K, \forall m \in \mathbb{N} .
$$

Theorem 1. Let $f \in \mathcal{E}(U)$ and suppose that condition $(*)$ is satisfied. Then $f \in C_{U}(M)$.

Proof. Let $K \subset U$ be a fixed compact set. For each $\xi \in \Omega$, we put

$$
\theta_{m}(\xi)=\sup _{x \in K} \frac{\left|\frac{d^{m}}{d t^{m}} f(x+t \xi)_{\mid t=0}\right|}{M(m)}, \quad m \in \mathbb{N}, \quad \theta(\xi)=\sup _{m \in \mathbb{N}} \theta_{m}(\xi) .
$$

Then $\theta$ is a lower semicontinuous function and by Baire's theorem, there exists an open subset $\Omega_{1} \subset \Omega$ and a constant $C_{1}>0$ such that

$$
\forall \xi \in \Omega_{1}, \quad \theta(\xi) \leq C_{1} .
$$


We have

$$
\frac{\partial^{m} f}{\partial \xi^{m}}(x):=\frac{d^{m}}{d t^{m}} f(x+t \xi)_{\mid t=0}=\sum_{|\omega|=m} D^{\omega} f(x) \frac{m !}{\omega_{1} ! \ldots \omega_{n} !} \xi_{1}^{\omega_{1}} \ldots \xi_{n}^{\omega_{n}}
$$

Since $\Omega_{1}$ is open in $S^{n-1}$, by a result of [6], there exists a constant $C_{2}>0$ such that

$$
\sup _{|\xi|=1}\left|\frac{\partial^{m} f}{\partial \xi^{m}}(x)\right| \leq C_{2}^{m} \sup _{\xi \in \Omega_{1}}\left|\frac{\partial^{m} f}{\partial \xi^{m}}(x)\right| \text {. }
$$

In view of Bernstein's inequality, there exists a constant $C_{3}>0$ such that

$$
C_{3}^{m} \sup _{|\omega|=m}\left|D^{\omega} f(x)\right| \leq \sup _{|\xi|=1}\left|\frac{\partial^{m} f}{\partial \xi^{m}}(x)\right| .
$$

Putting $\varrho=C_{2} / C_{3}$, we have

$$
\sup _{m} \sup _{\substack{x \in K \\|\omega|=m}} \frac{\left|D^{\omega} f(x)\right|}{M(m) \varrho^{m}}<\infty
$$

hence $f \in C_{U}(M)$.

REMARK 2. If $M(t)=t^{t}$, i.e. $m(t)=t \log t$, we have the analytic class. In the following we will consider $M$ such that the class $C_{U}(M)$ strictly contains the analytic class. We therefore take $m$ of the form

$$
m(t)=t \log t+t \mu(t)
$$

where $\mu$ is increasing and $\lim _{t \rightarrow \infty} \mu(t)=\infty$. In order to have $m^{\prime \prime}(t) \leq \delta$, we must suppose that $\mu(t) \leq a t$ for $t \gg 0(a>0)$. We also suppose that $\mu$ is in a Hardy field.

Proposition 1. $C_{U}(M)$ is closed under composition.

Proof. Since $t \mapsto t \mu(t)$ is convex, the proposition follows from [3].

Proposition 1 shows that we can define $C_{X}(M)$ by means of a local coordinate system when $X$ is a real-analytic manifold.

Let $t \mapsto M(t)$ be as above and for $s \in \mathbb{R}_{+}$, put

$$
\Lambda(s)=\inf _{t \geq t_{0}} M(t) s^{-t}
$$

where $t_{0}$ is a fixed positive real. The infimum is reached at a point $t$ where $m^{\prime}(t)=\log s$, and this point is unique since $m^{\prime}$ is increasing and $\lim _{t \rightarrow \infty} m^{\prime}(t)$ $=\infty$. We define $s \mapsto \omega(s)$ via $\Lambda(s)=e^{-\omega(s)}$. Then

or

$$
\left\{\begin{array}{l}
s=e^{m^{\prime}(t)} \\
\omega(s)=t m^{\prime}(t)-m(t)
\end{array}\right.
$$

$$
\left\{\begin{array}{l}
s=e t e^{\mu(t)+t \mu^{\prime}(t)} \\
\omega(s)=t+t^{2} \mu^{\prime}(t)
\end{array}\right.
$$


Since $\mu^{\prime}>0$, we have $\omega>0$ and $\lim _{t \rightarrow \infty} \omega(s)=\infty$. We can easily invert the last system to obtain

$$
\left\{\begin{array}{l}
t=s \omega^{\prime}(s), \\
m(t)=s \omega^{\prime}(s) \log s-\omega(s) .
\end{array}\right.
$$

Since $m(t)=t \log t+t \mu(t)$, we have

$$
\left\{\begin{array}{l}
t=s \omega^{\prime}(s) \\
\mu(t)=-\log \omega^{\prime}(s)-\frac{\omega(s)}{s \omega^{\prime}(s)} .
\end{array}\right.
$$

We see that $\omega$ is increasing and as $t \rightarrow \infty, s \omega^{\prime}(s) \rightarrow \infty$ and $-\log \omega^{\prime}(s)-$ $\omega(s) /\left(s \omega^{\prime}(s)\right) \rightarrow \infty$, so $\omega^{\prime}$ is decreasing and $\omega^{\prime}(s) \rightarrow 0$ as $s \rightarrow \infty$.

For $s>0$ let

$$
\lambda(s)=\inf _{n \in \mathbb{N}, n \geq t_{0}} M(n) s^{-n} .
$$

Lemma 3. For $s \gg 0$, we have

$$
e^{-\delta} \lambda(s) \leq \Lambda(s) \leq \lambda(s)
$$

Proof. Put $\alpha(t)=m(t)-t \log s$; we have $\Lambda(s)=e^{\alpha\left(t_{0}\right)}$ where $\alpha^{\prime}\left(t_{0}\right)=0$; then $\lambda(s)=e^{\alpha\left(n_{0}\right)}$ with $\left|n_{0}-t_{0}\right|<1$. Note that $\alpha\left(n_{0}\right)-\alpha\left(t_{0}\right)=\alpha^{\prime}\left((1-\theta) n_{0}\right.$ $\left.+\theta t_{0}\right), 0<\theta<1$. Since $m^{\prime \prime} \leq \delta$ and $\left|\alpha^{\prime}\left((1-\theta) n_{0}+\theta t_{0}\right)-\alpha\left(t_{0}\right)\right| \leq \delta$, we have $e^{-\delta} \lambda(s) \leq \Lambda(s)$. The second inequality is trivial.

Proposition 2. The following three statements are equivalent:

(i) $\sum_{n} \frac{M(n)}{M(n+1)}=\infty$,

(ii) $\int_{s_{0}}^{\infty} \frac{\omega(s)}{s^{2}} d s=\infty$ for some $s_{0}>0$,

(iii) $\int_{s_{0}}^{\infty} \frac{\log \lambda(s)}{s^{2}} d s=-\infty$ for some $s_{0}>0$,

Proof. We have $m^{\prime}(n) \leq m(n+1)-m(n) \leq m^{\prime}(n+1)$, hence

$$
\sum_{n} \frac{M(n)}{M(n+1)}=\infty \Leftrightarrow \int_{t_{0}}^{\infty} e^{-m^{\prime}(t)} d t=\infty .
$$

Recall that by the above,

$$
\int_{t_{0}}^{\infty} e^{-m^{\prime}(t)} d t=\int_{s_{0}}^{\infty} \frac{d\left(s \omega^{\prime}(s)\right)}{s} d s .
$$

Since $\omega^{\prime}(s) \rightarrow 0$ as $s \rightarrow \infty$ and it is decreasing, we have 


$$
\int_{s_{0}}^{\infty} \frac{d\left(s \omega^{\prime}(s)\right)}{s} d s=\infty \Leftrightarrow \int_{s_{0}}^{\infty} \frac{\omega(s)}{s^{2}} d s=\infty,
$$

which proves $($ i $) \Leftrightarrow\left(\right.$ ii). By Lemma 3, we have $-\omega(s) / s^{2} \leq(\log \lambda(s)) / s^{2}$, hence (ii) $\Leftrightarrow$ (iii).

Definition 2. We say that $C_{U}(M)$ is quasianalytic if for any $f \in C_{U}(M)$ and any $x \in U$ the Taylor series $T_{x} f$ of $f$ at $x$ uniquely determines $f$ around $x$.

By a well known result of Denjoy-Carleman, $C_{U}(M)$ is quasianalytic if and only if

$$
\sum_{n} \frac{M(n)}{M(n+1)}=\infty
$$

If the class is quasianalytic, Proposition 2 tells us that the function $\omega(s)$ tends to $\infty$ as $s \rightarrow \infty$ as rapidly as $s^{q}$, for all $q<1$. Probably the converse of this statement is also true.

In the case of the analytic class $(m(t)=t \log t)$, we have $\omega(s)=s \omega^{\prime}(s)$, hence $\omega(s)=C s$. The converse is also true:

Proposition 3. If $\omega(s) \simeq s$ as $s \rightarrow \infty$, then any $f \in C_{U}(M)$ is analytic.

Proof. By hypothesis, there exist $C>0$ and $A>0$ such that $\omega(s) \geq C s$ for all $s \geq A$; then

$$
\forall m \in \mathbb{N}, \forall s \geq A, \quad e^{-\omega(s)} \leq \frac{c^{-m}}{s^{m}} m ! .
$$

Since $m^{\prime}(t) \rightarrow \infty$ as $t \rightarrow \infty$, there exists $N_{0} \in \mathbb{N}$ such that $e^{m^{\prime}(t)} \geq A$ for all $t>N_{0}$ (we can suppose $\left.N_{0}>t_{0}\right)$. Let $r>N_{0}$ and put $s=e^{m^{\prime}(r)}$; then $s \geq A$ and $M(r) / s^{r} \leq \inf _{n \geq t_{0}} M(n) / s^{n}$. By Lemma 3, for all $m>N_{0}$ we have

$$
\frac{M(n)}{s^{n}} \leq e^{\delta} e^{-\omega(s)} \leq e^{\delta} \frac{C^{-m}}{s^{m}} m !,
$$

hence $M(m) \leq\left(e^{\delta} / C^{m}\right) m$ !. This proves the result.

Proposition 4. Let $\mu(t)=\log \log t$, i.e. $m(t)=t \log t+t \log \log t$. Then the class $C_{U}(M)$ is quasianalytic (recall that $\left.M(t)=e^{m(t)}\right)$.

Proof. We will show that $\int_{s_{0}}^{\infty}\left(\omega(s) / s^{2}\right) d s=\infty$. We have $s=e^{m^{\prime}(t)}=$ et $\log t e^{1 / \log t} \sim$ et $\log t$, and

Hence

$$
\omega(s)=t m^{\prime}(t)-m(t)=t+\frac{t}{\log t} \sim t \sim \frac{s}{e \log s} .
$$

$$
\frac{\omega(s)}{s^{2}} \sim \frac{1}{e s(\log s)} \quad \text { as } s \rightarrow \infty
$$

which proves the proposition. 
From now on we take $m(t)=t \log t+t \mu(t), \mu$ increasing, $\mu(t) \leq a t$ for $t \gg 0, a>0$, and $\lim _{t \rightarrow \infty} \mu(t)=\infty$. We also suppose that $\mu$ is in a Hardy field. Then the class $C_{U}(M)$ is an algebra, closed under differentiation and composition. We also take $\mu$ such that $C_{U}(M)$ is quasianalytic; for example, $\mu(t)=\log \log t$.

2.1. The ring of germs of quasianalytic functions. Let $r>0$. We use the notation $\Delta_{n}(r)=\left\{x \in \mathbb{R}^{n}|| x_{i} \mid<r\right.$ for $\left.1 \leq i \leq n\right\}$; if $x \in \mathbb{R}^{n}$, then $x=\left(x^{\prime}, x_{n}\right), x^{\prime} \in \mathbb{R}^{n-1} ;$ and we put $C_{n, r}(M)=C_{\Delta_{n}(r)}(M)$. If $f \in \mathcal{E}\left(\Delta_{n}(r)\right)$, we define, for $\varrho>0$,

$$
\|f\|_{\varrho, r, M}=\sup _{m} \sup _{\substack{|\alpha|=m \\ x \in \Delta_{n}(r)}} \frac{\left|D^{\alpha} f(x)\right|}{M(|\alpha|) \varrho^{|\alpha|}} \in[0, \infty]
$$

and we set $C_{n, \varrho, r}(M)=\left\{f \in C_{n, r}(M) \mid\|f\|_{\varrho, r, M}<\infty\right\}$. Clearly $C_{n, \varrho, r}(M)$ is a Banach space. Let $C_{n}(M)$ be the inductive limit of $C_{n, \varrho, r}(M)$ as $r \rightarrow 0$, $\varrho \rightarrow \infty$. We have an injection

$$
C_{n}(M) \rightarrow \mathbb{R}\left[\left[X_{1}, \ldots, X_{n}\right]\right]
$$

defined by $f \mapsto T_{0} f$.

In general, we will not distinguish notationally between the germ of a function and a representative of the germ.

Lemma 4. The algebra $C_{n}(M)$ is local and its maximal ideal is generated by $\left(x_{1}, \ldots, x_{n}\right)$.

Proof. Let $f \in C_{n}(M)$ be such that $f(0)=a_{0} \neq 0$; put $\varrho=\left|a_{0}\right|>0$ and $\varphi(\xi)=1 /\left(\xi+a_{0}\right)$. The function $\varphi$ is analytic in $\{\xi \in \mathbb{R}|| \xi \mid<\varrho\}$. Put $g=f-a_{0}$; then $g \in C_{n}(M)$ and $g(0)=0$. There exists $\eta>0$ such that $g\left([-\eta, \eta]^{n}\right) \subset\{\xi \in \mathbb{R}|| \xi \mid<\varrho\}$. By Proposition $1, \varphi \circ g \in C_{n}(M)$, hence $1 / f \in C_{n}(M)$. The algebra is then local and its maximal ideal is $\mathcal{M}=\left\{f \in C_{n}(M) \mid f(0)=0\right\}$.

Let $f \in \mathcal{M}$. Then $f(x)=\sum_{j=1}^{n} x_{j} g_{j}(x)$, where $g_{j}(x)=\int_{0}^{1} \frac{\partial f}{\partial x_{j}}(t x) d t$; we easily see that $g_{j} \in C_{n}(M)$ for $j=1, \ldots, n$.

Corollary 1. If $x^{\alpha}=x_{1}^{\alpha_{1}} \ldots x_{n}^{\alpha_{n}}$ divides $f \in C_{n}(M)$ in the ring of formal power series at $0 \in \mathbb{R}^{n}$, then $x^{\alpha}$ divides $f$ in $C_{n}(M)$.

Proof. This is an immediate consequence of the previous lemma.

Proposition 5. Let $f \in C_{n, \varrho_{1}, r_{1}}(M)-\{0\}$ be such that $f(0)=0$. For each $\varepsilon>0$, there exist $r^{\prime}, \varrho^{\prime}>0$ with $r^{\prime}<r_{1}$ and $\varrho^{\prime}>\varrho_{1}$ such that $\|f\|_{\varrho, r, M} \leq \varepsilon$ for all $r<r^{\prime}$ and $\varrho>\varrho^{\prime}$.

Proof. By hypothesis, we have 


$$
\sup _{m} \sup _{\substack{|\omega|=m \\ x \in \Delta_{n}\left(r_{1}\right)}} \frac{\left|D^{\omega} f(x)\right|}{M(|\omega|) \varrho_{1}^{m}}<\infty .
$$

Put

$$
R=\sup _{m \neq 0} \sup _{\substack{|\omega|=m \\ x \in \Delta_{n}\left(r_{1}\right)}} \frac{\left|D^{\omega} f(x)\right|}{M(|\omega|) \varrho_{1}^{m}} .
$$

Since $f(0)=0$ and $f \neq 0$, we have $R \neq 0$. Let $\varepsilon>0$ (we can suppose $\varepsilon<1$ ). There exists $\varrho^{\prime}>\varrho_{1}$ such that $\left(\varrho_{1} / \varrho\right)^{m} \leq \varepsilon / R$ for all $\varrho>\varrho^{\prime}$ and $m \in \mathbb{N}^{*}$. We then have

$$
\sup _{m \neq 0} \sup _{\substack{|\omega|=m \\ x \in \Delta_{n}\left(r_{1}\right)}} \frac{\left|D^{\omega} f(x)\right|}{M(|\omega|) \varrho^{m}} \leq \varepsilon .
$$

Since $f(0)=0$, there exists $r^{\prime}<r_{1}$ such that $|f(x)| \leq \varepsilon$ for all $r \leq r^{\prime}$ and $x \in \Delta_{n}(r)$. Hence $\|f\|_{\varrho, r, M} \leq \varepsilon$.

3. The implicit function theorem. It was proved in [7] that if the sequence $M(n)=M_{n}$ satisfies the conditions

$$
\begin{gathered}
\left(\frac{M_{q}}{q !}\right)^{1 /(q-1)} \leq C\left(\frac{M_{p}}{p !}\right)^{1 /(p-1)}, \quad 2 \leq q \leq p \\
M_{0}=M_{1}=1
\end{gathered}
$$

where $C>0$ is a constant, then the implicit function theorem holds in the ring $C_{n}(M)$.

Recall that $M(t)=e^{m(t)}, m(t)=t \log t+t \mu(t)$. We put $g(t)=t \mu(t)$. By Remark 1, we can suppose $M(1)=1$; we see that the condition (1) is satisfied if

$$
\forall p \geq q \geq 2, \quad(p-1) g(q) \leq C(q-1) g(p)
$$

for a constant $C>0$.

We remark that ( $\mu$ is increasing)

$$
\forall p \geq 1, \quad p g(p-1) \leq(p-1) g(p) .
$$

By repeating the process, we prove $(*)$. Hence the implicit function theorem holds in $C_{n}(M)$.

4. Algebraic properties. It is well known that the Weierstrass preparation theorem does not hold in $C_{n}(M)$ (see [3]). We do not know if $C_{n}(M)$ is a noetherian ring $(n>1)$. In this section we will show that $C_{n}(M)$ has a weak noetherian property which we call topological noetherianity. This property will be enough for us to extend some well known properties of semianalytic germs to the case where the germs are defined by equations and inequalities for elements in $C_{n}(M)$. 
We shall use a very elementary version of resolution of singularities consisting of blowings-up of a neighborhood of $0 \in \mathbb{R}^{n}, n>1$, say $V$, either with center an open subset, $W \subset \mathbb{R}^{n-p}, p<n$, such that $\{0\} \times W \subset V$, or with center $\{0\} \subset \mathbb{R}^{n}$.

4.1. Blowings-up. For each positive integer $r$, let $\mathbb{P}^{r-1}(\mathbb{R})$ denote the $(r-1)$-dimensional real projective space of lines through the origin in $\mathbb{R}^{r}$. Let $\sigma: \mathbb{R}^{r}-\{0\} \rightarrow \mathbb{P}^{r-1}(\mathbb{R})$ be the canonical surjection which associates to each $t \in \mathbb{R}^{r}-\{0\}$ the line, say $\sigma(t)$, in $\mathbb{R}^{r}$ passing through 0 and $t$. For each $i=1, \ldots, r$, let $V_{i}=\left\{x=\left(x_{1}, \ldots, x_{r}\right) \mid x_{i} \neq 0\right\}$ and $U_{i}=\sigma\left(V_{i}\right) ; U_{i}$ is a coordinate chart of $\mathbb{P}^{r-1}(\mathbb{R})$ with coordinates $\varphi_{i}: U_{i} \rightarrow \mathbb{R}^{r-1}$ given by

$$
\varphi_{i}(\sigma(t))=\left(\frac{t_{1}}{t_{i}}, \ldots, \frac{t_{i-1}}{t_{i}}, \frac{t_{i+1}}{t_{i}}, \ldots, \frac{t_{r}}{t_{i}}\right) .
$$

Definition 3. Let $V$ be an open neighborhood of 0 in $\mathbb{R}^{r}$. Put

$$
Z=\left\{(x, \sigma(t)) \in V \times \mathbb{P}^{r-1}(\mathbb{R}) \mid x \in \sigma(t)\right\}
$$

and let $\pi: Z \rightarrow V$ denote the mapping $\pi(x, \sigma(t))=x$. The mapping $\pi$ is called the blowing-up of $V$ with center 0 .

The mapping $\pi$ is proper, it restricts to a homeomorphism on $V-\{0\}$ and $\pi^{-1}(0)=\mathbb{P}^{r-1}(\mathbb{R})$.

We can cover $Z$ with coordinate charts

$$
Z_{i}=Z \cap V \times \sigma\left(U_{i}\right)
$$

with coordinates $\psi_{i}: Z_{i} \rightarrow \mathbb{R}^{r}$ given by

$$
\psi_{i}(x, \sigma(t))=\left(\frac{t_{1}}{t_{i}}, \ldots, \frac{t_{i-1}}{t_{i}}, x_{i}, \frac{t_{i+1}}{t_{i}}, \ldots, \frac{t_{r}}{t_{i}}\right) .
$$

In these local coordinates, $\pi$ is given by

$$
\pi\left(y_{1}, \ldots, y_{r}\right)=\left(y_{1} y_{i}, \ldots, y_{i-1} y_{i}, y_{i}, y_{i+1} y_{i}, \ldots, y_{r} y_{i}\right) .
$$

Let $n>r$ be an integer and $W$ an open subset of $\mathbb{R}^{n-r}=\left\{x=\left(x_{1}, \ldots, x_{n}\right) \in\right.$ $\left.\mathbb{R}^{n} \mid x_{1}=\ldots=x_{r}=0\right\}$. Let $w=\left(w_{1}, \ldots, w_{n-r}\right)$ be the coordinates of a point in $\mathbb{R}^{n-r}$. The mapping $\widetilde{\pi}=\pi \times \operatorname{id}_{W}: \widetilde{Z}=Z \times W \rightarrow V \times W$ is called the blowing-up of $V \times W$ with center $\{0\} \times W$. We can cover $\widetilde{Z}$ with coordinate charts

$$
\widetilde{Z}_{i}=\widetilde{Z} \cap V \times \sigma\left(U_{i}\right) \times W
$$

with coordinates $\widetilde{\varphi}_{i}: \widetilde{Z}_{i} \rightarrow V \times W$ given by

$$
\widetilde{\varphi}_{i}(x, \sigma(t), w)=\left(\frac{t_{1}}{t_{i}}, \ldots, \frac{t_{i-1}}{t_{i}}, x_{i}, \frac{t_{i+1}}{t_{i}}, \ldots, \frac{t_{r}}{t_{i}}, w\right) .
$$

We put $\widetilde{\varphi}_{i}=\left(y_{1}, \ldots, y_{r}, w^{\prime}\right)$.

Recall that $\mathcal{E}_{n}$ is the ring of germs at $0 \in \mathbb{R}^{n}$ of $C^{\infty}$ functions. Let $a \in \widetilde{\pi}^{-1}(0) \cap \widetilde{Z}_{i}$ and $f \in \mathcal{E}_{n}$; then the Taylor expansion of $f \circ \widetilde{\pi}$ at $a$ is given by 
formal substitution of $w=w^{\prime}, X_{i}=y_{i}$, and $X_{l}=y_{i}\left(y_{l}(a)+y_{l}\right), l \neq i$, in the Taylor expansion of $f$ at 0 . In particular if $\widehat{f} \in \mathbb{R}[[X, W]]$ is a formal series, we will denote by $\widehat{f} \circ \widehat{\pi}_{a}$ the formal series obtained by formal substitution of $w=w^{\prime}, X_{i}=y_{i}$, and $X_{l}=y_{i}\left(y_{l}(a)+y_{l}\right), l \neq i$, in the formal series $\widehat{f}$.

We need the following lemma proved in [10]; for completeness we will give the proof.

Lemma 5. Let $\Omega \subset \mathbb{N}^{n}, n>1$, be a finite set and put $\mathcal{F}=\left\{X^{\alpha}=\right.$ $\left.X_{1}^{\alpha_{1}} \ldots X_{n}^{\alpha_{n}} \mid \alpha=\left(\alpha_{1}, \ldots, \alpha_{n}\right) \in \Omega\right\}$. Let $V$ be an open neighborhood of 0 in $\mathbb{R}^{n}$. Then there exists a real-analytic manifold $Z$ and a proper real-analytic surjective mapping $\pi: Z \rightarrow V$ such that:

(a) For all $a \in \pi^{-1}(0)$ there is a chart $U$ with $a \in U$ and with coordinates $y=\left(y_{1}, \ldots, y_{n}\right)$ such that the set $\left\{\mu_{\alpha} \in \mathbb{N}^{n} \mid X^{\alpha} \circ \widehat{\pi}_{a}=y^{\mu_{\alpha}}\right\}$ is totally ordered by the product order on $\mathbb{N}^{n}$.

(b) $\pi_{\mid U}: U \rightarrow V$ is the composition of a finite sequence of blowings-up.

Proof. We proceed by induction on $n \geq 2$. We can suppose that the cardinality of $\Omega$ is 2. After making a finite number of blowings-up of $V$ with center the origin of $\mathbb{R}^{2}$, we can easily see that the lemma is true for $n=2$. Suppose $n \geq 3$. After dividing each monomial by the common factors, we can also suppose that there is $r \in \mathbb{N}, r \leq n$, such that the monomials are of the form $X_{1}^{\alpha_{1}} \ldots X_{r}^{\alpha_{r}}$ or $X_{r+1}^{\alpha_{r+1}} \ldots X_{n}^{\alpha_{n}}$ with $\alpha_{n}=\min _{i=1, \ldots, n} \alpha_{i}$ (after making a permutation of $\left.\left(X_{1}, \ldots, X_{n}\right)\right)$.

We proceed by induction on $\alpha_{n}$. If $\alpha_{n}=0$ we are done by the inductive hypothesis on $n$. Suppose $\alpha_{n}>0$ and consider the two monomials $A=$ $X_{1}^{\alpha_{1}} \ldots X_{r}^{\alpha_{r}}$ and $B=X_{r+1}^{\alpha_{r+1}} \ldots X_{n-1}^{\alpha_{n-1}}$; by the induction hypothesis on $n$, if $V^{\prime}$ is a neighborhood of $0 \in \mathbb{R}^{n-1}$, there exist a real-analytic manifold $M$ and a proper real-analytic surjective mapping $\pi: M \rightarrow V^{\prime}$ such that conditions (a) and (b) of the lemma are satisfied. Let $a \in \pi^{-1}(0)$. There is a chart $U^{\prime}$ with $a \in U^{\prime}$ and with coordinates $y=\left(y_{1}, \ldots, y_{n-1}\right)$ such that $A \circ \widehat{\pi}_{a}=$ $y_{1}^{\beta_{1}} \ldots y_{n-1}^{\beta_{n-1}}$ and $B \circ \widehat{\pi}_{a}=y_{1}^{\beta_{1}^{\prime}} \ldots y_{n-1}^{\beta_{n-1}^{\prime}}$ with $\left(\beta_{1}, \ldots, \beta_{n-1}\right) \leq\left(\beta_{1}^{\prime}, \ldots, \beta_{n-1}^{\prime}\right)$ or $\left(\beta_{1}^{\prime}, \ldots, \beta_{n-1}^{\prime}\right) \leq\left(\beta_{1}, \ldots, \beta_{n-1}\right)$. Consider the two monomials $y_{1}^{\beta_{1}} \ldots y_{n-1}^{\beta_{n-1}}$ and $y_{1}^{\beta_{1}^{\prime}} \ldots y_{n-1}^{\beta_{n-1}^{\prime}} X_{n}^{\alpha_{n}}$ on $U^{\prime} \times \mathbb{R}$. If $\left(\beta_{1}, \ldots, \beta_{n-1}\right) \leq\left(\beta_{1}^{\prime}, \ldots, \beta_{n-1}^{\prime}\right)$ we are done. Suppose $\left(\beta_{1}^{\prime}, \ldots, \beta_{n-1}^{\prime}\right)<\left(\beta_{1}, \ldots, \beta_{n-1}\right)$; after dividing by common factors, we are in the situation of $y_{1}^{\gamma_{1}} \ldots y_{n-1}^{\gamma_{n-1}}$ and $X_{n}^{\alpha_{n}}$. If $\gamma_{i}<\alpha_{n}$ for some $i$, then we use the second induction (on $\alpha_{n}$ ). Suppose $\gamma_{i} \geq \alpha_{n}$ for all $i=1, \ldots, n-1$. We will blow up $U^{\prime} \times \mathbb{R}$ with center $y_{1}=X_{n}=0$. Let $\widetilde{\pi}: \widetilde{U} \rightarrow U^{\prime} \times \mathbb{R}$ be this blowing-up. We can cover $\widetilde{U}$ by two coordinate charts: $\widetilde{U}_{1}$ and $\widetilde{U}_{2}$. With respect to these charts, $\widetilde{\pi}$ is given, respectively, by

$$
\tilde{\pi}\left(y_{1}, \ldots, y_{n}\right)=\left(y_{1}, y_{2}, \ldots, y_{n-1}, y_{n} y_{1}\right)
$$

and 


$$
\widetilde{\pi}\left(y_{1}, \ldots, y_{n}\right)=\left(y_{n} y_{1}, y_{2}, \ldots, y_{n-1}, y_{n}\right)
$$

In the chart $\widetilde{U}_{1}$ our monomials are of the form

$$
y_{1}^{\gamma_{1}-\alpha_{n}} y_{2}^{\gamma_{2}} \ldots y_{n-1}^{\gamma_{n-1}}, \quad y_{n}^{\alpha_{n}}
$$

By continuing, we will have $\gamma_{1}-\alpha_{n}<\alpha_{n}$ and the inductive hypothesis on inf $\gamma_{i}$ will prove the lemma. In the second chart $\widetilde{U}_{2}$, the result is true since $\left(\gamma_{1}, \ldots, \gamma_{n-1}, \gamma_{1},\right) \geq\left(0, \ldots, 0, \alpha_{n}\right)$.

Proposition 6. Let $\widehat{f} \in \mathbb{R}\left[\left[X_{1}, \ldots, X_{n}\right]\right]$ and let $V \subset \mathbb{R}^{n}$ be an open neighborhood of 0 . There exists a real-analytic manifold $Z$ and a proper real-analytic surjective mapping $\pi: Z \rightarrow V$ such that each $a \in \pi^{-1}(0)$ admits a coordinate neighborhood $U$ with coordinates $y=\left(y_{1}, \ldots, y_{n}\right)$ such that $\widehat{f} \circ \widehat{\pi}_{a}=y_{1}^{\alpha_{1}} \ldots y_{n}^{\alpha_{n}} \widehat{h}$, where $\widehat{h} \in \mathbb{R}\left[\left[Y_{1}, \ldots, Y_{n}\right]\right]$ is a unit.

Proof. Let us remark that we can write $\widehat{f}$ in the form

$$
\widehat{f}=\sum_{\omega \in \Omega \subset \mathbb{N}^{n}} \widehat{f}_{\omega} X^{\omega}
$$

where $\Omega \subset \mathbb{N}^{n}$ is finite and $\widehat{f}_{\omega} \in \mathbb{R}\left[\left[X_{1}, \ldots, X_{n}\right]\right]$ is a unit for each $\omega \in \Omega$. By Lemma 5 there exists a real-analytic manifold $Z$ and a real-analytic proper surjective mapping $\pi: Z \rightarrow V$ such that each $a \in \pi^{-1}(0)$ admits a coordinate neighborhood $U$ with coordinates $y=\left(y_{1}, \ldots, y_{n}\right)$ and the set $\left\{\mu_{\omega} \mid X^{\omega} \circ \widehat{\pi}_{a}=y^{\mu_{\omega}}\right\}$ is totally ordered. Let $\mu_{\omega_{0}}$ be the least element. We have

$$
\widehat{f} \circ \widehat{\pi}_{a}=\sum_{\omega \in \Omega \subset \mathbb{N}^{n}} \widehat{f}_{\omega} \circ \widehat{\pi}_{a} X^{\omega} \circ \widehat{\pi}_{a}=y^{\mu_{\omega_{0}}} \sum_{\omega \in \Omega \subset \mathbb{N}^{n}} \widehat{f}_{\omega} \circ \widehat{\pi}_{a} y^{\mu_{\omega}-\mu_{\omega_{0}}} .
$$

This proves the result.

Proposition 7. Let $f \in C_{n}(M)$. Then there exists an open neighborhood $V$ of $0 \in \mathbb{R}^{n}$, a real-analytic manifold $Z$ and a proper real-analytic surjective mapping $\pi: Z \rightarrow V$ such that each $a \in \pi^{-1}(0)$ admits a coordinate neighborhood $U$ with coordinates $y=\left(y_{1}, \ldots, y_{n}\right)$ such that $f \circ \pi_{\mid U}(y)=$ $y^{\mu} \varphi(y)$, where $\mu \in \mathbb{N}^{n}, \varphi \in C_{U}(M)$ and $\varphi(y) \neq 0$ for all $y \in U$.

Proof. Choose an open neighborhood $V$ of $0 \in \mathbb{R}^{n}$ where $f$ is defined and Proposition 6 can be applied. Then there exists a real-analytic manifold $Z$ and a proper real-analytic surjective mapping $\pi: Z \rightarrow V$ such that each $a \in \pi^{-1}(0)$ admits a coordinate neighborhood $U$ with coordinates $y=\left(y_{1}, \ldots, y_{n}\right)$ in which $T_{0} f \circ \widehat{\pi}_{a}=y^{\mu} \widehat{h}$, where $\widehat{h} \in \mathbb{R}\left[\left[y_{1}, \ldots, y_{n}\right]\right]$ is a unit. Since $f \circ \pi_{\mid U} \in C_{U}(M)$, Corollary 1 implies that $f \circ \pi_{\mid U}=y^{\mu} \varphi(y), \varphi(0) \neq 0$, which proves the proposition.

\section{Topological noetherianity}

LEMMA 6. Every decreasing sequence of germs $f_{1}^{-1}(0) \supset f_{2}^{-1}(0) \supset \ldots$ with $f_{j} \in C_{n}(M)$ is stationary. 
Proof. By induction on $n$; the lemma is trivially true for $n=1$. Suppose $n>1$ and the result holds for $n-1$. According to Proposition 7 , there exists an open neighborhood $V$ of $0 \in \mathbb{R}^{n}$, a real-analytic manifold $Z$ and a proper real-analytic surjective mapping $\pi: Z \rightarrow V$ such that each $a \in \pi^{-1}(0)$ admits a coordinate neighborhood $U$ with coordinates $y=\left(y_{1}, \ldots, y_{n}\right)$ in which $f_{1} \circ \pi_{\mid U}(y)=y^{\mu} \varphi(y)$ and $\varphi(y) \neq 0$ for all $y \in U$. It is enough to prove that the sequence $\left(f_{j} \circ \pi\right)^{-1}(0)$ is stationary in a neighborhood of every point $a \in \pi^{-1}(0)$. We can suppose that $f_{1}(y)=y_{1}^{\mu_{1}} \ldots y_{n}^{\mu_{n}} \varphi(y)$ and $\varphi(y) \neq 0$ for all $y \in U$. Let $J=\left\{j=1, \ldots, n \mid \mu_{j} \neq 0\right\}$. For each $j \in J$ the sequence $\left(f_{l}^{-1}(0) \cap\left\{y \in U \mid y_{j}=0\right\}\right)_{l}$ is stationary by the inductive hypothesis; so our sequence is stationary near $a$, which proves the lemma.

\subsection{M-manifolds}

Definition 4. An $n$-dimensional manifold is a Hausdorff space with countable basis in which each point has a neighborhood homeomorphic to an open set in $\mathbb{R}^{n}$. An $M$-structure on a manifold $Z$ is a family $\mathcal{F}=\left\{\left(U_{i}, \varphi_{i}\right) \mid\right.$ $i \in I\}$ of homeomorphisms $\varphi_{i}$, called local coordinate systems, of an open set $U_{i} \subset Z$ onto an open set $\widetilde{U}_{i} \subset \mathbb{R}^{n}$ such that:

(a) If $\left(U_{i}, \varphi_{i}\right),\left(U_{j}, \varphi_{j}\right) \in \mathcal{F}$, then each cartesian component of the map $\varphi_{j} \circ \varphi_{i}^{-1}: \varphi_{i}\left(U_{i} \cap U_{j}\right) \subset \mathbb{R}^{n} \rightarrow \varphi_{j}\left(U_{i} \cap U_{j}\right) \subset \mathbb{R}^{n}$ is in $C_{\varphi_{i}\left(U_{i} \cap U_{j}\right)}(M)$.

(b) $Z=\bigcup_{i \in I} U_{i}$.

A manifold with an $M$-structure is called an $M$-manifold.

Let $Z$ be an $M$-manifold and $U \subset Z$ an open set. A function $\varphi$ defined in $U$ will be said to be in $C_{U}(M)$ if for every coordinate system $\left(U_{i}, \varphi_{i}\right)$, the composite $\varphi \circ \varphi_{i}^{-1}$ is in $C_{\varphi_{i}\left(U_{i} \cap U\right)}(M)$. We shall sometimes denote $\varphi \circ \varphi_{i}^{-1}$ by $\varphi_{\mid U_{i} \cap U}$.

Let us remark that every real-analytic manifold is an $M$-manifold.

Let $Y \subset Z$. We say that $Y$ is a smooth $M$-submanifold if $Y$ is covered by coordinate charts $U$ of $M$, each of which has local coordinates $z=(x, y)$, $x=\left(x_{1}, \ldots, x_{m}\right), y=\left(y_{1}, \ldots, y_{p}\right)$, in which $Y \cap U=\left\{y_{1}=\ldots=y_{p}=0\right\}$.

Let $Z$ be an $M$-manifold and $Y$ a closed $M$-submanifold of $Z$. We define the blowing-up $\pi: Z^{\prime} \rightarrow Z$ with center $Y$ as follows: $Z^{\prime}$ is an $M$-manifold and $\pi$ is a proper map in the class $M$ such that:

(1) $\pi$ restricts to an isomorphism $Z^{\prime}-\pi^{-1}(Y) \rightarrow Z-Y$ in the class $M$.

(2) Let $U \subset Z$ be a coordinate chart with local coordinates in $U$ defined by $\varphi: U \rightarrow V \times W$, where $U, W$ are open neighborhoods of the origin in $\mathbb{R}^{p}, \mathbb{R}^{n-p}$, respectively, and $\varphi(U \cap Y)=\{0\} \times W$. Let $\pi_{0}: V^{\prime} \rightarrow V$ be the blowing-up of $V$ with center $\{0\}$. Then there is an isomorphism $\varphi^{\prime}: \pi^{-1}(U) \rightarrow V^{\prime} \times W$ in the class $M$ such that

$$
\pi_{0} \times \operatorname{id}_{W} \circ \varphi^{\prime}=\varphi \circ \pi_{\mid \pi^{-1}(U)} .
$$


Definition 5. Let $Z$ be an $M$-manifold. Let $U$ be an open subset of $Z$ and let $Y$ be a closed $M$-submanifold of $U$. Let $\pi: Z^{\prime} \rightarrow Z$ denote the composition of the blowing-up $Z^{\prime} \rightarrow U$ of $U$ with center $Y$ and the inclusion $U \rightarrow Z$. We call $\pi$ a local blowing-up of $Z$ with center $Y$.

We will consider mappings $\pi: Z^{\prime} \rightarrow Z$ obtained as the composition of a finite sequence of local blowings-up; i.e. $\pi=\pi_{1} \circ \ldots \circ \pi_{k}$, where, for each $i=1, \ldots, k, \pi_{i}: Z_{i+1} \rightarrow Z_{i}$ is a local blowing-up of $Z_{i}$, and $Z_{1}=Z$, $Z_{k+1}=Z^{\prime}$

6. Łojasiewicz's inequality. In the following, $Z$ will be an $M$-manifold with $\operatorname{dim} Z=n$ and $W$ an open subset of $Z$. As an immediate consequence of Proposition 7, we have:

Proposition 8. Let $f \in C_{W}(M)$. Then each $a \in W$ admits an open neighborhood $V$ for which there exists an $M$-manifold $Z^{\prime}$ and a proper surjective mapping $\pi: Z^{\prime} \rightarrow V$ in the class $M$ such that:

(i) each $b \in \pi^{-1}(a)$ admits a coordinate neighborhood $U$ with coordinates $y=\left(y_{1}, \ldots, y_{n}\right)$ in which $f \circ \pi(y)=y^{\mu} \varphi(y)$ for all $y \in U$, where $\varphi \in C_{U}(M)$ and $\varphi(y) \neq 0$ for all $y \in U$.

(ii) $\pi_{\mid U}: U \rightarrow V$ is a finite composition of local blowings-up.

REMARK 3. We require that the mapping $\pi: Z^{\prime} \rightarrow V$ satisfy the following additional condition: each $b \in \pi^{-1}(a)$ admits a coordinate neighborhood $U_{b}$ for which there exists $q \in \mathbb{N}$ and an isomorphism $\varphi^{\prime}: U_{b} \rightarrow \varphi^{\prime}\left(U_{b}\right) \subset$ $V \times \mathbb{P}^{q}(\mathbb{R})$ in the class $M$ such that $\varphi^{\prime}\left(U_{b}\right)$ is an $M$-submanifold defined by homogeneous polynomial equations (in homogeneous coordinates of $\mathbb{P}^{q}(\mathbb{R})$ ) whose coefficients are in $C_{V}(M)$.

A local blowing-up has this property. We can easily see that the composition of two local blowings-up also has this property. By condition (ii) of the last proposition, we see that $\pi$ can be chosen as in the remark.

Theorem 2. Let $f \in C_{W}(M)$ and set $V_{W}(f)=\{x \in W \mid f(x)=0\}$. Let $g$ be any $C^{\infty}$ function on $W$ such that $g(x)=0$ for all $x \in V_{W}(f)$. Then, for every compact subset $K \subset W$, there exist $N, C>0$ such that

$$
|g(x)|^{N} \leq C|f(x)|, \quad \forall x \in K .
$$

Proof. We can suppose that $|g(x)| \leq 1$ for all $x \in K$. The question is local in $W$, so we will prove that each $a \in W$ admits a coordinate neighborhood $V_{a}$ for which there exist $N_{a}, C_{a}>0$ such that

$$
|g(x)|^{N_{a}} \leq C_{a}|f(x)|, \quad \forall x \in V_{a} .
$$

Then we can cover $K$ by finite $V_{a_{i}}, i=1, \ldots, l$, and take $N=\max _{i} N_{a_{i}}$, $C=\max _{i} C_{a_{i}}$. 
Let $a \in W$. By Proposition 8, there exists a coordinate neighborhood $V_{a}$ of $a$ with coordinates $x=\left(x_{1}, \ldots, x_{n}\right)$ centered at $a$, i.e. $x_{i}(a)=0$ for all $i=1, \ldots, n$, an $M$-manifold $Z^{\prime}$ and a proper surjective mapping $\pi: Z^{\prime} \rightarrow V_{a}$ in the class $M$ such that

$(*)$ each $b \in \pi^{-1}(a)$ admits a coordinate neighborhood $U_{b}$ with coordinates $y=\left(y_{1}, \ldots, y_{n}\right)$ centered at $b$ in which $f \circ \pi(y)=y^{\mu} \varphi(y)$ for all $y \in U_{b}$, where $\mu \in \mathbb{N}^{n}, \varphi \in C_{U_{b}}(M)$ and $\varphi(y) \neq 0$ for all $y \in U_{b}$. Since $\pi$ is proper, there exists a finite set $\Lambda \subset \mathbb{N}$ such that $\bigcup_{\alpha \in \Lambda} U_{b_{\alpha}}$ is an open covering of $\pi^{-1}(a), b_{\alpha} \in \pi^{-1}(a)$ for all $\alpha \in \Lambda$ and $f \circ \pi(y)=y^{\mu_{\alpha}} \varphi_{\alpha}(y)$ for all $y \in U_{b_{\alpha}}, \varphi_{\alpha}(y) \neq 0$, for all $y \in U_{b_{\alpha}}$ and $\varphi_{\alpha} \in C_{U_{b_{\alpha}}}(M)$.

Write $\mu_{\alpha}=\left(\mu_{\alpha 1}, \ldots, \mu_{\alpha n}\right)$ and let $\Delta_{\alpha}$ be the set of those $i$ where $\mu_{\alpha i}>0\left(\Delta_{\alpha}\right.$ may be empty for some $\alpha$ ). The assumption on $g$ implies that $g \circ \pi$ vanishes identically on each of the hyperplanes $y_{i}=0$ in $U_{b_{\alpha}}$ with $i \in \Delta_{\alpha}$. Hence $g \circ \pi$ is divisible by the product of those $y_{i}$ with $i \in \Delta_{\alpha}$. Then $g \circ \pi(y)=y^{\beta_{\alpha}} h_{\alpha}(y)$ for all $y \in U_{b_{\alpha}}, \beta_{\alpha}=\left(\beta_{\alpha 1}, \ldots, \beta_{\alpha n}\right)$ and $h_{\alpha}$ is a $C^{\infty}$ function on $U_{b_{\alpha}}$. Recall that $\beta_{\alpha j}>0$ if $j \in \Delta_{\alpha}$.

Let $\Delta_{\alpha}^{\prime}=\left\{j \in \Delta_{\alpha} \mid \beta_{\alpha j}<\mu_{\alpha j}\right\}$ and put $q_{\alpha}=\max _{j \in \Delta_{\alpha}^{\prime}} \mu_{\alpha j} / \beta_{\alpha j}$. We see that $(g \circ \pi(y))^{q_{\alpha}}=\psi_{\alpha}(y)(f \circ \pi)(y)$ for all $y \in U_{b_{\alpha}}$, where $\psi_{\alpha}$ is a $C^{\infty}$ function on $U_{b_{\alpha}}$.

If $r>0$, we write $U_{b_{\alpha}}(r):=\left\{y \in U_{b_{\alpha}} \mid \sum_{i=1}^{n} y_{i}^{2} \leq r\right\}$; since $\pi$ is proper, there exists $\varrho>0$ such that

$$
V_{a}(\varrho)=\left\{x \in V_{a} \mid \sum_{i=1}^{n} x_{i}^{2} \leq \varrho\right\} \subset \bigcup_{\alpha \in \Lambda} \pi\left(U_{b_{\alpha}}(\varrho)\right) .
$$

Let $C_{\alpha}=\sup _{y \in U_{b_{\alpha}}(\varrho)}\left|\psi_{\alpha}(y)\right|, C=\max C_{\alpha}$ and $N=\max q_{\alpha}$. Then for all $x \in V_{a}(\varrho)$, we have $|g(x)|^{N} \leq|f(x)|$, which proves the theorem.

Let us remark that by the previous proof the infimum of $\lambda>0$ such that there exists $C>0$ with $|g(x)|^{\lambda} \leq C|f(x)|$ for all $x \in K$ is a rational number.

Theorem 3. Suppose that $W \subset \mathbb{R}^{n}$ is an open set and $f \in C_{W}(M)$. Then for each compact subset $K \subset W$, we can find $N, C>0$ such that

$$
C|f(x)| \geq d\left(x, V_{W}(f)\right)^{N}, \quad \forall x \in K .
$$

Proof. We will prove that each $a \in W$ admits a neighborhood $V_{a}$ and constants $N_{a}, C_{a}>0$ such that

$$
C_{a}|f(x)| \geq d\left(x, V_{V_{a}}(f)\right)^{N_{a}}, \quad \forall x \in V_{a} .
$$

Let $a \in W$. There exists $\pi: Z^{\prime} \rightarrow V_{a}$ as in the proof of the previous theorem. We then have a finite covering of $\pi^{-1}(a) \subset \bigcup_{\alpha \in \Lambda} U_{b_{\alpha}}$ and for all $\alpha \in \Lambda, f \circ \pi(y)=y^{\mu_{\alpha}} \varphi_{\alpha}(y)$ for all $y \in U_{b_{\alpha}}$. Then $V_{U_{b_{\alpha}}}(f \circ \pi)$ is equal to the 
union of those coordinate hyperplanes $H_{\alpha i}$ defined by $y_{i}$ with $i \in \Delta_{\alpha}$. Define $\psi_{\alpha i}(y)=d\left(\pi(y), \pi \gamma_{\alpha i}(y)\right)^{2}$ for $y \in U_{b_{\alpha}}$, where $\gamma_{\alpha i}(y)$ denotes the orthogonal projection from $U_{b_{\alpha}} \simeq \mathbb{R}^{n}$ to $H_{\alpha i}$. We see that $\psi_{\alpha i}$ is a $C^{\infty}$ function on $U_{b_{\alpha}}$. Let $\psi_{\alpha}=\prod_{i \in \Delta_{\alpha}} \psi_{\alpha i}$. Then:

- $\psi_{\alpha}$ is a $C^{\infty}$ function on $U_{b_{\alpha}}$,

- $\psi_{\alpha}(y) \geq d\left(\pi(y), V_{V_{a}}(f)\right)^{2 n_{\alpha}}$, where $n_{\alpha}$ is the number of elements of $\Delta_{\alpha}$. We have $V_{U_{b_{\alpha}}}(f \circ \pi) \subset V_{U_{b_{\alpha}}}\left(\psi_{\alpha}\right)$; by the previous theorem, there exist $\varrho, N_{\alpha}, C_{\alpha}>0$ such that for all $y \in U_{b_{\alpha}}(\varrho)$,

$$
C_{\alpha}|(f \circ \pi)(y)| \geq\left|\psi_{\alpha}(y)\right|^{N_{\alpha}} .
$$

Let $N=\max _{\alpha \in \Lambda} N_{\alpha} /\left(2 n_{\alpha}\right)$ with $n_{\alpha} \neq 0$. Then for all $x \in V_{a}(\varrho)$ we have $C|f(x)| \geq d\left(x, V_{V_{a}(\varrho)}\right)^{N}$, where $C=\max _{\alpha \in \Lambda} C_{\alpha}$.

\section{Quasi semianalytic sets}

Definition 6. Let $A$ be a subset of an $M$-manifold $Z$. Then $A$ is said to be quasi semianalytic at $a \in Z$ if there exists an open neighborhood $V$ of $a$ in $Z$ and a finite number of elements of $C_{V}(M), g_{i}$ and $f_{i j}$, such that

$$
A \cap V=\bigcup_{i}\left\{x \in V \mid g_{i}(x)=0, f_{i j}(x)>0, \forall j\right\} .
$$

If $A$ is quasi semianalytic at every point of $Z$, we say that $A$ is quasi semianalytic in $Z$.

REMARK 4. (i) The property of being quasi semianalytic is preserved under locally finite unions, locally finite intersections and complements.

(ii) If $A \subset Z$ is a quasi semianalytic set it is easy to see that for all $a \in Z$, there exists an open neighborhood $V$ of $a$ in $Z$ such that $A \cap V$ is a finite disjoint union of sets of the form

$$
\left\{x \in V \mid \varphi_{0}(x)=0, \varphi_{1}(x)>0, \ldots, \varphi_{r}(x)>0\right\},
$$

where $\varphi_{0}, \varphi_{1}, \ldots, \varphi_{r}$ are in $C_{V}(M)$.

TheOrem 4. Let $A$ be a quasi semianalytic set in $Z$. Then each $x \in Z$ admits a neighborhood $V$ such that $A \cap V$ has only a finite number of connected components.

Proof. We will use the notation of Theorem 2 with $f=\prod_{i, j} g_{i} f_{i j}$. It is enough to prove that for each $\alpha \in \Lambda$, the number of connected components of $U_{b_{\alpha}} \cap \pi^{-1}(A)$ is finite. Since $f \circ \pi(y)=y^{\mu_{\alpha}} \varphi_{\alpha}(y)$ and $\varphi_{\alpha}(y) \neq 0$ for all $y \in U_{b_{\alpha}}$, we can easily see that

$$
g_{i} \circ \pi(y)=y^{\mu_{\alpha_{i}}} \varphi_{\alpha_{i}}(y), \quad f_{i j} \circ \pi(y)=y^{\mu_{\alpha_{i j}}} \varphi_{\alpha_{i j}}(y), \quad \forall y \in U_{b_{\alpha}},
$$

where $\varphi_{\alpha_{i}}(y) \neq 0$ and $\varphi_{\alpha_{i j}}(y) \neq 0$ for all $y \in U_{b_{\alpha}}$ and all $i, j$. This shows that $U_{b_{\alpha}} \cap \pi^{-1}(A)$ has only a finite number of connected components, which proves the theorem. 
Let us give some notations and definitions. Let $U$ be an open subset of $Z$, and $A \subset U$. We define $I_{U}(A):=\left\{f \in C_{U}(M) \mid f(x)=0, \forall x \in A\right\}$; it is an ideal of $C_{U}(M)$. Let $F \subset U$; we say that $F$ is a global quasianalytic set in $U$ if there exist $h_{1}, \ldots, h_{q} \in C_{U}(M)$ such that $F=\left\{x \in U \mid h_{1}(x)=0\right.$, $\left.\ldots, h_{q}(x)=0\right\}$. Suppose that $U$ is a chart of $Z, a \in U$, with coordinates $x=\left(x_{1}, \ldots, x_{n}\right)$ centered at $a$. If $f \in C_{U}(M)$ we denote by $\nu_{a}(f)$ the maximum of $q \in \mathbb{N}$ such that the Taylor expansion of $f$ at $a, T_{a} f$, is in $\underline{m}^{q}$ ( $\underline{m}$ is the maximal ideal of $\left.\mathbb{R}\left[\left[X_{1}, \ldots, X_{n}\right]\right]\right)$.

Proposition 9. Let $F$ be a global quasianalytic set in $U$. Let $k \in \mathbb{N}$ be the maximum of the integers such that there exist $f_{1}, \ldots, f_{k} \in I_{U}(F)$ with jacobian $\Delta=\frac{D\left(f_{1}, \ldots, f_{k}\right)}{D\left(x_{i_{1}}, \ldots, x_{i_{k}}\right)} \notin I_{U}(F)$. Put $\Gamma=\left\{x \in U \mid f_{1}(x)=\ldots=f_{k}(x)\right.$ $=0, \Delta(x) \neq 0\}$. Then $F-V(\Delta):=\{x \in F \mid \Delta(x) \neq 0\}$ is a submanifold of $U$, and is quasi semianalytic; moreover $F-V(\Delta)$ is the union of some connected components of $\Gamma$.

Proof. Clearly we have $F-V(\Delta) \subset \Gamma$. In order to prove the proposition, it is enough to prove that for each $x \in F-V(\Delta)$, the germs of $\Gamma$ and $F-V(\Delta)$ at $x$ are the same. We will prove that the germ of $\Gamma$ at $x, \Gamma_{x}$, is contained in $(F-V(\Delta))_{x}$. Suppose, for a contradiction, that $\Gamma_{x} \not \subset(F-$ $V(\Delta))_{x}$; then there exists $g \in I_{U}(F)$ such that $g_{\mid \Gamma_{x}} \neq 0$. By Lemma 7 below, there exists $h \in\{1, \ldots, n\}-\left\{i_{1}, \ldots, i_{k}\right\}$ such that if $g_{1}=\frac{D\left(f_{1}, \ldots, f_{k}, g\right)}{D\left(x_{i_{1}}, \ldots, x_{i_{k}}, x_{h}\right)} \mid \Gamma_{x}$, then $\nu_{x}\left(g_{1}\right)<\nu_{x}\left(g_{\mid \Gamma_{x}}\right)$. By definition of $k$, we have $g_{1} \in I_{U}(F)$ and also $g_{1 \mid \Gamma_{x}} \neq 0$. We continue with $g_{1}$ in place of $g$ and so on. At the end we find $g_{q} \in I_{U}(F)$ with $g_{q}(x) \neq 0$, which is a contradiction.

Lemma 7. Let $U$ be an open neighborhood of 0 in $\mathbb{R}^{n}$, and put

$$
S=\left\{x \in U \mid f_{1}(x)=\ldots=f_{k}(x)=0, \Delta(x)=\frac{D\left(f_{1}, \ldots, f_{k}\right)}{D\left(x_{1}, \ldots, x_{k}\right)}(x) \neq 0\right\},
$$

where $f_{1}, \ldots, f_{k} \in C_{U}(M)$. Suppose that $0 \in S$. Let $g \in C_{U}(M)$ be such that $g_{\mid S} \neq 0$. Then there exists $h$ with $k<h \leq n$ such that

$$
\left.\nu_{0}\left(g_{\mid S}\right)>\nu_{0}\left[\frac{D\left(f_{1}, \ldots, f_{k}, g\right)}{D\left(x_{1}, \ldots, x_{k}, x_{h}\right)}\right)_{\mid S}\right] .
$$

Proof. Since the mapping

$$
x=\left(x_{1}, \ldots, x_{n}\right) \mapsto\left(f_{1}(x), \ldots, f_{k}(x), x_{k+1}, \ldots, x_{n}\right)
$$

is a local diffeomorphism near 0 , we can suppose that $f_{i}(x)=x_{i}$ for all $i=1, \ldots, k$. The result is then obvious.

In the following we call $\Gamma=\left\{x \in U \mid f_{1}(x)=\ldots=f_{k}(x)=0, \Delta(x) \neq 0\right\}$ a quasianalytic stratum. Let $B \subset U ; B$ is called a quasi semianalytic stratum 
if $B$ is the intersection of a quasi analytic stratum with an open set of the form $\left\{x \in U \mid \varphi_{1}(x)>0, \ldots, \varphi_{q}(x)>0\right\}$, where $\varphi_{1}, \ldots, \varphi_{q} \in C_{U}(M)$.

Let $U \subset Z$ be a chart of $Z$ with coordinates $y=\left(y_{1}, \ldots, y_{n}\right)$. Let $B \subset U$. We say that $B$ is a quadrant if $B$ is defined by a system of some equalities $y_{i}=0$ and some inequalities $\varepsilon_{j} y_{j}>0$ with $\varepsilon_{j}= \pm 1$.

Theorem 5. Let $A \subset Z$ be a quasi semianalytic set. Then each $a \in Z$ admits an open neighborhood $V$ such that $A \cap V=\bigcup_{j=1}^{s} \Lambda_{j}$, where each $\Lambda_{j}$ is a submanifold of $V, \Lambda_{i} \cap \Lambda_{j}=\emptyset$ if $i \neq j$, and $\Lambda_{j}$ is a finite union of connected components of a quasi semianalytic stratum.

Proof. By Remark 4(ii), one can assume $A=\left\{x \in U \mid \varphi_{0}(x)=0\right.$, $\left.\varphi_{1}(x)>0, \ldots, \varphi_{q}(x)>0\right\}$, where $\varphi_{0}, \ldots, \varphi_{q} \in C_{U}(M)$ and $U$ is an open neighborhood of $a$ in $Z$. Let $F=\left\{x \in U \mid \varphi_{0}(x)=0\right\}$. By Proposition 9, there exists $f_{0} \in C_{U}(M), f_{0} \notin I_{U}(F)$, such that the set $F-V\left(f_{0}\right)=$ $\left\{x \in F \mid f_{0}(x) \neq 0\right\}$ is the union of some connected components of a quasinalytic stratum. Put $F_{1}=\left\{x \in U \mid \varphi_{0}^{2}(x)+f_{0}^{2}(x)=0\right\}$; then $F_{1} \subset F$. We repeat the same thing with $F_{1}$ in place of $F$. Thus we construct a decreasing sequence $F \supset F_{1} \supset \ldots$, where $F_{j}=V\left(f_{j}\right), f_{j} \in C_{U}(M)$, such that for each $j \in \mathbb{N}, F_{j}-F_{j+1}$ is the union of some connected components of a quasianalytic stratum. By Lemma 6 , there exists $s \in \mathbb{N}$ and an open neighborhood $V$ of $a$ such that $F_{j} \cap V=F_{j+1} \cap V$ for all $j>s$. For $j \leq s$, put $\widetilde{\Gamma}_{j}=F_{j}-F_{j+1}$; then $V \cap F=\bigcup_{j=1}^{s} \widetilde{\Gamma}_{j} \cap V$. We then see that

$$
A \cap V=\bigcup_{j=1}^{s} \Lambda_{j},
$$

where $\Lambda_{j}=\left\{x \in \widetilde{\Gamma}_{j} \cap V \mid \varphi_{1}(x)>0, \ldots, \varphi_{q}(x)>0\right\}$. By shrinking $V$ if necessary, we see that $\Lambda_{j}$ has a finite number of connected components (Theorem 4), which proves the theorem.

By the previous theorem, we define the topological dimension of $A$ at $a \in Z, \operatorname{dim}_{a} A$, to be the maximum of the dimensions of $\Lambda_{j}, j=1, \ldots, s$. This definition is independent of the family $\Lambda_{j}: \operatorname{dim}_{a} A=q$ if and only if $A$ contains an open set homeomorphic to an open ball in $\mathbb{R}^{q}$, but not an open set homeomorphic to an open ball in $\mathbb{R}^{l}, l>q$.

TheOREm 6. Let $A \subset Z$ be a quasi semianalytic set. Then each connected component of $A$ is a quasi semianalytic set. The closure of $A$ in $Z$, $\bar{A}$, is also a quasi semianalytic set.

Proof. Let $\Gamma \subset A$ be a connected component of $A$. Let $a \in Z$ be such that the germ of $\Gamma$ at $a$ is not empty. There exists a neighborhood $V_{a}$ of $a$ in $Z$ such that $A \cap V_{a}$ is a finite union of sets of the form 


$$
\Lambda=\left\{x \in V_{a} \mid \varphi_{0}(x)=0, \varphi_{1}(x)>0, \ldots, \varphi_{q}(x)>0\right\},
$$

where $\varphi_{0}, \varphi_{1}, \ldots, \varphi_{q} \in C_{V_{a}}(M)$.

Clearly we can suppose that $A \cap V=\Lambda$. Let $f=\varphi_{0} \varphi_{1} \ldots \varphi_{q}$. We keep the notation of the proof of Theorem 2. Since $\pi^{-1}(\Gamma) \cap U_{b_{\alpha}}$ is open and closed in $\pi^{-1}(A) \cap U_{b_{\alpha}}$, it follows that $\pi^{-1}(\Gamma) \cap U_{b_{\alpha}}$ is a finite union of quadrants in $U_{b_{\alpha}}$. By Remark 3, there exists $q \in \mathbb{N}$ such that $U_{b_{\alpha}}$ is isomorphic to an $M$-submanifold of $V_{a} \times \mathbb{P}^{q}(\mathbb{R})$ defined by homogeneous polynomials with coefficients in $C_{V_{a}}(M)$. By Lemma 8 below, $\pi\left(\pi^{-1}(\Gamma) \cap U_{b_{\alpha}}\right)$ is a quasi semianalytic set. Since $\pi$ is proper, there exists a neighborhood $V_{a}^{\prime} \subset V_{a}$ of $a$ such that $\pi^{-1}\left(V_{a}^{\prime}\right) \subset \bigcup_{\alpha \in \Lambda} U_{b_{\alpha}}$; then $\pi\left[\bigcup_{\alpha \in \Lambda} U_{b_{\alpha}}\right]$ is a neighborhood of $a$ ( $\pi$ is surjective) and $\bigcup_{\alpha} \pi\left(U_{b_{\alpha}}\right) \cap \Gamma=\bigcup_{\alpha} \pi\left(\pi^{-1}(\Gamma) \cap U_{b_{\alpha}}\right)$, which proves the first statement.

We can choose, for each $\alpha \in \Lambda$, a closed neighborhood $U_{b_{\alpha}}^{\prime} \subset U_{b_{\alpha}}$ of $a$ such that $\pi^{-1}(a) \subset \bigcup_{\alpha} U_{b_{\alpha}}^{\prime}$. Let

$$
A_{1}=\bigcup_{\alpha} \pi\left(U_{b_{\alpha}}^{\prime} \cap \overline{\pi^{-1}(A)}\right) .
$$

We have $A_{1} \subset V_{a} \cap \bar{A}$ and $V_{a}^{\prime} \cap \bar{A} \subset A_{1}$.

Now since $U_{b_{\alpha}}^{\prime} \cap \overline{\pi^{-1}(A)}=\overline{\pi^{-1}(A) \cap U_{b_{\alpha}}^{\prime}}$, and $\pi^{-1}(A) \cap U_{b_{\alpha}}^{\prime}$ is a finite union of quadrants, by Lemma $8, \pi\left(U_{b_{\alpha}}^{\prime} \cap \overline{\pi^{-1}(A)}\right)$ is a quasi semianalytic set, hence so is $V_{a} \cap \bar{A}$ since it coincides with $A_{1}$ in a neighborhood of $a$ (namely, in $V_{a}^{\prime}$ ).

It remains to recall Łojasiewicz's version of the Tarski-Seidenberg theorem.

Lemma 8 ([8]). Let $U \subset Z$ be an open set. Put

$$
\begin{aligned}
& A=\bigcup_{i=1}^{s}\left\{\left(x, t_{1}, \ldots, t_{q}\right) \in U \times \mathbb{R}^{q} \mid g_{i}\left(x, t_{1}, \ldots, t_{q}\right)=0,\right. \\
&\left.f_{i, 1}\left(x, t_{1}, \ldots, t_{q}\right)>0, \ldots, f_{i, r}\left(x, t_{1}, \ldots, t_{q}\right)>0\right\},
\end{aligned}
$$

where $g_{i}, f_{i, j} \in C_{U}(M)\left[t_{1}, \ldots, t_{q}\right]$ for all $i, j$. If $\pi: U \times \mathbb{R}^{q} \rightarrow U$ denotes the projection, then $\pi(A)$ is a quasi semianalytic set.

8. Quasi subanalytic sets. Let $U \subset \mathbb{R}^{2}$ be an open neighborhood of the origin and $\varphi: U \subset \mathbb{R}^{2} \rightarrow \mathbb{R}^{3}$ a mapping with components $\varphi_{1}, \varphi_{2}, \varphi_{3} \in$ $C_{U}(M)$. We suppose that there are no nontrivial formal relations between the Taylor series $T_{0} \varphi_{1}, T_{0} \varphi_{2}, T_{0} \varphi_{3}$ of $\varphi_{1}, \varphi_{2}, \varphi_{3}$ at the origin. Let $r>0$ be such that $W=\left\{(x, y) \in \mathbb{R}^{2} \mid x^{2}+y^{2} \leq r\right\} \subset U$. Then $A=\varphi(W) \subset \mathbb{R}^{3}$ is not quasi semianalytic at the origin in $\mathbb{R}^{3}$, whereas $A$ is the projection of the set $\left\{\left(x, y, t_{1}, t_{2}, t_{3}\right) \in U \times \mathbb{R}^{3} \mid x^{2}+y^{2} \leq r, t_{i}=\varphi_{i}(x, y), i=1,2,3\right\}$ which is a relatively compact quasi semianalytic set. 
Thus the Tarski-Seidenberg theorem is false for quasi semianalytic sets.

Definition 7. Let $Z$ be an $M$-manifold and $A \subset Z$. We say that $A$ is quasi subanalytic in $Z$ if for each $a \in Z$, there exists an open neighborhood $U$ of $a$ in $Z$, an $M$-manifold $Z^{\prime}$ and a relatively compact quasi semianalytic set $A \subset Z \times Z^{\prime}$ in $Z \times Z^{\prime}$ such that $\pi(A)=A \cap U$, where $\pi: Z \times Z^{\prime} \rightarrow Z$ is the projection.

From the properties of quasi semianalytic sets, we can easily see that a locally finite union and intersection of quasi subanalytic sets is quasi subanalytic. The closure and each connected component of a quasi subanalytic set are quasi subnalytic; a projection of a relatively compact quasi subanalytic set is quasi subanalytic.

We will prove that the complement (and thus the interior) of a quasi subanalytic set is quasi subanalytic. First, we establish some measure properties of a quasi subanalytic set. By the work of Charbonnel [2] and Wilkie [12], we will first show that we have a uniform bound on the number of connected components of the fibers of a projection restricted to a relatively compact quasi subanalytic set; more precisely:

TheOREM 7. Let $Z$ and $Z^{\prime}$ be two $M$-manifolds and $A$ be a relatively compact quasi subanalytic set in $Z \times Z^{\prime}$. Let $\pi: Z \times Z^{\prime} \rightarrow Z$ be the projection. Then the number of connected components of a fiber $\pi^{-1}(x) \cap A$ is bounded uniformly in $x \in Z$.

Proof. We proceed by induction on $\operatorname{dim} Z$. If $\operatorname{dim} Z=0$, the result is true, since $A$ is relatively compact. Suppose that $n:=\operatorname{dim} Z \geq 1$ and the result is true for $n-1$. We can assume that $Z=\mathbb{R}^{n}, Z^{\prime}=\mathbb{R}^{p}$ and $A$ is relatively compact and quasi semianalytic in $\mathbb{R}^{n} \times \mathbb{R}^{p}$. We argue by induction on the maximum dimension of the fibers $A_{x}=\pi^{-1}(x) \cap A$ for $x \in \mathbb{R}^{n}$. By Lemma 6 , it is enough to find a quasianalytic set $F \subset \mathbb{R}^{n} \times \mathbb{R}^{p}$ such that the assertion is true for $A-F$. By Theorem 5 , we can suppose that $A$ is a connected component of a quasi semianalytic stratum

$$
\begin{aligned}
S=\left\{(x, y) \in \mathbb{R}^{n} \times \mathbb{R}^{p} \mid f_{1}(x, y)\right. & =\ldots=f_{k}(x, y)=0, \\
\delta(x, y) & \left.\neq 0, g_{1}(x, y)>0, \ldots, g_{q}(x, y)>0\right\},
\end{aligned}
$$

where $\delta(x, y)$ is the jacobian of $\left(f_{1}, \ldots, f_{k}\right)$. Let $n-\beta, 0 \leq \beta \leq n$, be the maximum rank of $\pi_{\mid S}$. Then there exists a jacobian

$$
\delta_{1}(x, y)=\frac{D\left(f_{1}, \ldots, f_{k}\right)}{D\left(x_{i_{1}}, \ldots, x_{i_{\beta}}, y_{j_{1}}, \ldots, y_{j_{\alpha}}\right)}
$$

with $\alpha+\beta=k$ such that $\delta_{1} \notin I(S)$. We take $F=\left\{(x, y) \in \mathbb{R}^{n} \times \mathbb{R}^{p}\right.$ $\left.\delta_{1}(x, y)=0\right\}$ and put $S^{\prime}=S-F$. The rank of $\pi_{\mid S^{\prime}}: S^{\prime} \rightarrow \mathbb{R}^{n}$ is constant and equal to $n-\beta$. For all $x \in \mathbb{R}^{n}, S_{x}^{\prime}=\pi^{-1}(x) \cap S^{\prime}$ is a submanifold of dimension $p-\alpha$. 
We can suppose, for the proof, that $p-\alpha=0$. Indeed, if $p-\alpha \geq 1$, then each connected component, say $C$, of $\pi^{-1}(x) \cap S^{\prime}$ satisfies $\bar{C}-C \neq \emptyset$ (the projection $\pi^{-1}(x) \cap S^{\prime} \rightarrow\left\{y \in \mathbb{R}^{p} \mid y_{j_{1}}=\ldots=y_{j_{\alpha}}=0\right\}$ is open). Let $\psi(x, y)=$ $\sum_{j=1}^{q} g_{j}(x, y)+\delta(x, y)^{2}+\delta_{1}(x, y)^{2}$; then $\psi(x, y)>0$ on $C$ and $\psi(x, y)=0$ if $(x, y) \in \bar{C}-C$. Put $S^{\prime \prime}=\left\{(x, y) \mid \operatorname{grad}\left(\psi_{\mid \pi^{-1}(x) \cap S^{\prime}}\right)(x, y)=0\right\}$. Then $S^{\prime \prime}$ is a quasi semianalytic set. Since $\psi$ is not constant on any connected component of $\pi^{-1}(x) \cap S^{\prime}$, we have $\operatorname{dim} S_{x}^{\prime \prime}<\operatorname{dim} S_{x}^{\prime}$ for all $x \in \mathbb{R}^{n}\left(S_{x}^{\prime \prime}=S^{\prime \prime} \cap \pi^{-1}(x)\right)$. We remark that $\psi$ has a positive maximum on each connected component of $\pi^{-1}(x) \cap S^{\prime}$, hence $S_{x}^{\prime \prime} \neq \emptyset$. By the inductive hypothesis on the dimension of the fibers, the theorem is true for $S^{\prime \prime}$, which implies the result for $S^{\prime}$.

Suppose $p-\alpha=0$. Then $S_{x}^{\prime}$ is a finite set for all $x \in \mathbb{R}^{n}$. We consider two cases:

CASE 1: $n-\beta<n$. Let $\pi_{1}: \mathbb{R}^{n} \rightarrow \mathbb{R}^{n-\beta}=\left\{x \in \mathbb{R}^{n} \mid x_{i_{1}}=\ldots=\right.$ $\left.x_{i_{\beta}}=0\right\}$ be the projection. The inductive hypothesis on $n$ implies that the assertion is true for the mapping $\pi_{1} \circ \pi_{\mid S^{\prime}}$, and hence for $\pi_{\mid S^{\prime}}: S^{\prime} \rightarrow \mathbb{R}^{n}$.

CASE 2: $n-\beta=n$. Let $\pi^{\prime}: \mathbb{R}^{n} \rightarrow \mathbb{R}^{n-1}$ be the projection onto $x_{n}=0$ and put $\widetilde{\pi}=\pi^{\prime} \circ \pi$. Then $\widetilde{\pi}_{\mid S^{\prime}}: S^{\prime} \rightarrow \mathbb{R}^{n-1}$ is a submersion. For all $x^{\prime} \in \mathbb{R}^{n-1}$, $\tilde{\pi}^{-1}\left(x^{\prime}\right) \cap S^{\prime}$ is the disjoint union of a finite number of connected curves of class $M$; by the inductive hypothesis on $n$, the number of these curves is bounded when $x^{\prime} \in \mathbb{R}^{n-1}$. In order to prove that the number of points in $\pi^{-1}(x) \cap S^{\prime}$ is bounded $\left(x=\left(x^{\prime}, x_{n}\right)\right)$, we will prove that no connected component of $\widetilde{\pi}^{-1}\left(x^{\prime}\right) \cap S^{\prime}$ contains two points of $\pi^{-1}(x) \cap S^{\prime}$, which proves our result, since the number of connected components of $\widetilde{\pi}^{-1}\left(x^{\prime}\right) \cap S^{\prime}$ is bounded when $x^{\prime} \in \mathbb{R}^{n-1}$.

Suppose, for a contradiction, that there exists a connected component $C$ of $\widetilde{\pi}^{-1}\left(x^{\prime}\right) \cap S^{\prime}$ which contains $a, b \in \pi^{-1}(x) \cap S^{\prime}, a \neq b$. The curve $C$ intersects $\pi^{-1}(x)$ in two points $a, b$. By the generalized Rolle lemma [5], there exists $\xi \in C$ such that the tangent space to $C$ at $\xi$ contains a parallel vector to $\pi^{-1}(x)=\mathbb{R}^{n}$. Hence the tangent space $T_{\xi} S^{\prime}$ contains a vector parallel to $\pi^{-1}(x)=\mathbb{R}^{n}$, which is a contradiction since $T_{\xi} S^{\prime}$ is transverse to $\mathbb{R}^{n}$.

Definition 8. Let $Z$ be an $M$-manifold and $A \subset Z$. We say that $A$ is Lebesgue measurable [resp. A has measure zero] if for any coordinate chart $U$ with coordinates $\varphi=\left(x_{1}, \ldots, x_{n}\right), \varphi(U \cap A)$ is Lebesgue measurable in $\mathbb{R}^{n}$ [resp. $\varphi(U \cap A)$ has measure zero].

Using the last theorem and properties of the class of quasi subanalytic sets cited above, we prove:

THEOREM 8. Let $A$ be a quasi subanalytic set. The following conditions are equivalent:

(1) A has no interior point. 
(2) $\bar{A}$ has no interior point.

(3) A has measure zero.

(4) $\bar{A}$ has measure zero.

Proof. The proof uses Theorem 7 and is the same as in [9].

Definition 9. Let $Z^{\prime}$ be an $M$-manifold. A mapping $f: A \subset Z \rightarrow Z^{\prime}$ is quasi subanalytic if its graph $\Gamma_{f}$ is quasi subanalytic in $Z \times Z^{\prime}$.

We will use the following result:

Proposition 10 ([9]). If $f: A \subset Z \rightarrow Z^{\prime}$ is a quasi subanalytic mapping, then the set of points in $A$ where $f$ is not continuous has no interior points.

In the following we will show that the dimension of a quasi semianalytic set is well behaved.

Lemma 9. If $A \subset Z$ is a nonempty quasi semianalytic set, then we have $\operatorname{dim}(\bar{A}-A)<\operatorname{dim} A$.

Proof. Recall that, by Theorem $6, \bar{A}-A$ is quasi semianalytic. Suppose, for a contradiction, that $\operatorname{dim}(\bar{A}-A)=: n-k \geq \operatorname{dim} A=: n-l$. We can suppose that $Z=\mathbb{R}^{n}$ and $A$ is relatively compact. Let $\Lambda$ be a connected component of a quasi semianalytic stratum $S \subset \mathbb{R}^{n}$ such that $\Lambda \subset \bar{A}-A$ and $\operatorname{dim} \Lambda=\operatorname{dim}(\bar{A}-A)$. We have

$$
\begin{aligned}
S=\left\{x \in \mathbb{R}^{n} \mid\right. & f_{1}(x)=\ldots=f_{k}(x)=0, \\
& \left.\delta(x)=\frac{D\left(f_{1}, \ldots, f_{k}\right)}{D\left(x_{i_{1}}, \ldots, x_{i_{k}}\right)}(x) \neq 0, g_{1}(x)>0, \ldots, g_{q}(x)>0\right\} ;
\end{aligned}
$$

note that $k \leq l$ by hypothesis.

Let $\pi_{n-k}: \mathbb{R}^{n} \rightarrow \mathbb{R}^{n-k}=\left\{x \in \mathbb{R}^{n} \mid x_{i_{1}}=\ldots=x_{i_{k}}=0\right\}$ be the projection; then $\pi_{n-k \mid \Lambda}: \Lambda \rightarrow \mathbb{R}^{n-k}$ is a local diffeomorphism. Let $a \in \Lambda$ and put $a^{\prime}=\pi_{n-k}(a)$. There exist balls $B_{n}(a, r)$ and $B_{n-k}\left(a^{\prime}, r\right)$ in $\mathbb{R}^{n}$ and $\mathbb{R}^{n-k}$ respectively such that $\pi_{n-k \mid \Lambda \cap B_{n}(a, r)}: \Lambda \cap B_{n}(a, r) \rightarrow B_{n-k}\left(a^{\prime}, r\right)$ is a diffeomorphism; let $g: B_{n-k}\left(a^{\prime}, r\right) \rightarrow \Lambda \cap B_{n}(a, r)$ be the inverse.

Let $B=\left\{x^{\prime} \in B_{n-k}\left(a^{\prime}, r\right) \mid \exists x \in A \cap B_{n}(a, r), \pi_{n-k}(x)=x^{\prime}\right\}$. Then $B$ is a quasi subanalytic set. Clearly, we have $B_{n-k}\left(a^{\prime}, r / 2\right) \subset \bar{B}$; hence, by Theorem $8, \operatorname{int}(B) \neq \emptyset$; this implies that $k=l$. Put $\pi_{k}: \mathbb{R}^{n} \rightarrow \mathbb{R}^{k}=$ $\left\{x \in \mathbb{R}^{n} \mid x_{j}=0, \forall j \notin\left\{i_{1}, \ldots, i_{k}\right\}\right\}$. For each $p=1,2, \ldots$, let

$$
B_{p}=\left\{x^{\prime} \in B \mid \exists y_{1}, \ldots, y_{p} \in \mathbb{R}^{k}, y_{i} \neq y_{j} \text { if } i \neq j, y_{i} \in \pi_{k}\left[A \cap \pi_{n-k}^{-1}\left(x^{\prime}\right)\right]\right\} .
$$

We have

$$
\ldots \subset B_{\nu+1} \subset B_{\nu} \subset \ldots \subset B_{2} \subset B_{1}=B .
$$

By Theorem 7 , there exists $\mu \in \mathbb{N}^{*}$ such that $\operatorname{int}\left(B_{\mu}\right) \neq \emptyset$ and $\operatorname{int}\left(B_{\mu+1}\right)=\emptyset$. We then have $\operatorname{int}\left(\bar{B}_{\mu+1}\right)=\emptyset$, hence $\operatorname{int}\left(B_{\mu}\right) \cap B-\bar{B}_{\mu+1} \neq \emptyset$. Thus there exists a ball $B^{\prime} \subset B_{\mu}-B_{\mu+1}$. For each $x^{\prime} \in B^{\prime}, \pi_{k}^{-1}\left(x^{\prime}\right) \cap A$ contains exactly 
$\mu$ elements, so we can construct $\mu$ functions $h_{1}, \ldots, h_{\mu}: B^{\prime} \subset \mathbb{R}^{n-k} \rightarrow \mathbb{R}^{k}$ such that $\Gamma_{h_{j}}$ is quasi subanalytic for all $j=1, \ldots, \mu$, and $\pi_{k}\left[A \cap \pi_{n-k}^{-1}\left(x^{\prime}\right)\right]=$ $\left\{h_{1}\left(x^{\prime}\right), \ldots, h_{\mu}\left(x^{\prime}\right)\right\}$ for all $x^{\prime} \in B^{\prime}$. By construction, $h_{j}\left(x^{\prime}\right) \neq \pi_{k} \circ g\left(x^{\prime}\right)$ for all $j=1, \ldots, \mu$ and $x^{\prime} \in B^{\prime}$.

By Proposition 10, there is a ball $B^{\prime \prime} \subset B$ such that the restrictions of all $h_{1}, \ldots, h_{\mu}$ are continuous on $B^{\prime \prime}$ and there exists $c>0$ such that $\left|h_{j}\left(x^{\prime}\right)-\pi_{k}\left(g\left(x^{\prime}\right)\right)\right|>c$ for all $x^{\prime} \in B^{\prime \prime}$ and $j=1, \ldots, \mu$; but this contradicts the fact that $g\left(x^{\prime}\right) \in \Lambda \subset \bar{A}-A$ for all $x^{\prime} \in B^{\prime \prime}$; hence the lemma.

\section{Theorem of the complement}

Theorem 9. Let $Z$ be an $M$-manifold and let $B \subset Z$ be a quasi subanalytic set. Then $Z-B$ is quasi subanalytic.

Proof. We can assume that $Z=\mathbb{R}^{n}$ and $B$ is relatively compact. We argue by induction on $n$. There exists a relatively compact semianalytic set $A \subset \mathbb{R}^{n} \times \mathbb{R}^{p}$ such that $\pi(A)=B$, where $\pi: \mathbb{R}^{n} \times \mathbb{R}^{p} \rightarrow \mathbb{R}^{n}$ is the projection. By Theorem 5 , we can assume that $A$ is a connected component of a quasi semianalytic stratum

$$
\begin{aligned}
S=\left\{(x, y) \in \mathbb{R}^{n} \times \mathbb{R}^{p} \mid\right. & f_{1}(x, y)=\ldots=f_{k}(x, y)=0, \\
\delta(x, y) & \left.\neq 0, g_{1}(x, y)>0, \ldots, g_{q}(x, y)>0\right\} .
\end{aligned}
$$

As in the proof of Theorem 7 (and with the same notations), it is enough to find a quasianalytic set $F \subset \mathbb{R}^{n} \times \mathbb{R}^{p}$ such that $A-F \neq \emptyset$ and the assertion is true for $\pi(A-F)$. We take $F$ as in the proof of Theorem 7 and put $A^{\prime}=A-F \subset S^{\prime}=S-F$. We proceed by induction on the maximum dimension of the fibers $\pi^{-1}(x) \cap A^{\prime}$. Recall that $\operatorname{dim}\left(\pi^{-1}(x) \cap S^{\prime}\right)=p-\alpha$ for all $x \in \mathbb{R}^{n}$.

Suppose that $p-\alpha=0$; then $\operatorname{dim} S^{\prime}=n-\beta \leq n$. We consider two cases:

CASE 1: $\beta>0$. Let $\pi_{1}: \mathbb{R}^{n} \rightarrow \mathbb{R}^{n-\beta}=\left\{x \in \mathbb{R}^{n} \mid x_{i_{1}}=\ldots=x_{i_{\beta}}=0\right\}$ be the projection. The inductive hypothesis shows that the theorem is true in $\mathbb{R}^{n-\beta}$. Put $\pi^{\prime}=\pi_{1} \circ \pi$. The number of points in $S^{\prime} \cap \pi^{\prime-1}(u)$ is bounded when $u \in \mathbb{R}^{n-\beta}$. Therefore the number of points in $\pi\left(A^{\prime}\right) \cap \pi_{1}^{-1}(u)$ is bounded. By Lemma 10 below, the complement of $\pi\left(A^{\prime}\right)$ in $\mathbb{R}^{n}$ is quasi subanalytic.

CASE 2: $\beta=0$. We then have $\operatorname{dim} S^{\prime}=n$. Let $Q=\overline{A^{\prime}}-A^{\prime} ;$ by Lemma 9 , $\operatorname{dim} Q<n$, hence, by the first case, $\mathbb{R}^{n}-\pi(Q)$ is quasi subanalytic. We have $\mathbb{R}^{n}-\pi\left(A^{\prime}\right)=\left(\mathbb{R}^{n}-\pi\left(\overline{A^{\prime}}\right) \cup\left(\pi(Q)-\pi\left(A^{\prime}\right) \cap \pi(Q)\right)\right.$. By case $1, \mathbb{R}^{n}-\pi\left(A^{\prime}\right) \cap \pi(Q)$ is quasi subanalytic, hence $\mathbb{R}^{n}-\pi\left(A^{\prime}\right)$ is quasi subanalytic.

If $p-\alpha>0$, we see that there exists $S^{\prime \prime} \subset S^{\prime}$ such that $\operatorname{dim} S^{\prime \prime}<$ $\operatorname{dim} S^{\prime}, S^{\prime \prime}$ is quasi semianalytic and $\pi\left(S^{\prime \prime}\right)=\pi\left(S^{\prime}\right)$. By using the inductive hypothesis on the maximum dimension of the fibers $\pi^{-1}(x) \cap A^{\prime}$, we deduce that $\mathbb{R}^{n}-\pi\left(A^{\prime}\right)$ is quasi subanalytic. 
Lemma 10. Suppose that, in $\mathbb{R}^{n}$, the complement of every quasi subanalytic set is quasi analytic. Let $A \subset \mathbb{R}^{n} \times \mathbb{R}^{p}$ be a relatively compact quasi subanalytic set. Suppose that the number of points in the fibers $A \cap \pi^{-1}(x), x \in$ $\mathbb{R}^{n}$, is bounded, where $\pi: \mathbb{R}^{n} \times \mathbb{R}^{p} \rightarrow \mathbb{R}^{n}$ is the projection. Then $\mathbb{R}^{n} \times \mathbb{R}^{p}-A$ is quasi subanalytic.

Proof. The proof is the same as in [1, Lemma 3.9].

\section{References}

[1] E. Bierstone and P. D. Milman, Semianalytic and subanalytic sets, Publ. Math. I.H.E.S. 67 (1989), 5-42.

[2] J.-Y. Charbonnel, Sur certains sous-ensembles de l'espace euclidien, Ann. Inst. Fourier (Grenoble) 41 (1991), no. 3, 679-717.

[3] C. L. Childress, Weierstrass division in quasianalytic local rings, Canad. J. Math. 28 (1976), 938-953.

[4] E. M. Dyn'kin, Pseudoanalytic extension of smooth functions, Amer. Math. Soc. Transl. (2) 115 (1980), 33-58.

[5] A. G. Khovanskiū, Real analytic varieties with the finiteness property and complex abelian integrals, Funct. Anal. Appl. 18 (1984), 199-207.

[6] M. Klimek, Pluripotential Theory, London Math. Soc. Monogr. 6, Clarendon Press, 1991.

[7] H. Komatsu, The implicit function theorem for ultradifferentiable mappings, Proc. Japan Acad. Ser. A 55 (1979), 69-72.

[8] S. Łojasiewicz, Ensembles semi-analytiques, preprint, École Polytechnique, Paris, 1965.

[9] S. Maxwell, A general model completeness result for expansions of the real ordered field, Ann. Pure Appl. Logic 95 (1998), 185-227.

[10] J.-P. Rolin, P. Speissegger and A. J. Wilkie, Quasianalytic Denjoy-Carleman classes and o-minimality, J. Amer. Math. Soc. 16 (2003), 751-777.

[11] J.-C. Tougeron, Sur les ensembles semi-analytiques avec conditions Gevrey au bord, Ann. Sci. École Norm. Sup. 27 (1994), 173-208.

[12] A. J. Wilkie, A theorem of the complement and some new o-minimal structures, Selecta Math. 5 (1999), 397-421.

Faculty of Sciences

Department of Mathematics

B.P. 133, Kénitra, 14000, Morocco

E-mail: kabdelhafed@hotmail.com

Received January 13, 2003

Revised version June 13, 2003 